\title{
Effect of ethnicity on care pathway and outcomes in patients hospitalized with influenza A(H1N1)pdm09 in the UK
}

\author{
G. A. NYLAND ${ }^{1} \dagger$, B. C. MCKENZIE ${ }^{*} \dagger$, P. R. MYLES ${ }^{1}$, M. G. SEMPLE ${ }^{2}$, \\ W. S. LIM ${ }^{3}$, P. J. M. OPENSHAW ${ }^{4}$, R. C. READ ${ }^{5}$, B. L. TAYLOR ${ }^{6}$, S. J. BRETT ${ }^{7}$, \\ J. MCMENAMIN ${ }^{8}$, J. E. ENSTONE ${ }^{1}$, B. BANNISTER ${ }^{9,10}$, K. G. NICHOLSON ${ }^{11}$ \\ AND J.S. NGUYEN-VAN-TAM ${ }^{1}$ on behalf of the Influenza Clinical Information Network \\ (FLU-CIN) \\ ${ }^{1}$ University of Nottingham, Nottingham, UK; ${ }^{2}$ University of Liverpool, Liverpool, UK; ${ }^{3}$ Nottingham University \\ Hospitals NHS Trust, Nottingham, UK; ${ }^{4}$ Imperial College, London, UK; ${ }^{5}$ University of Southampton, \\ Southampton, UK; ${ }^{6}$ Portsmouth Hospitals NHS Trust, Portsmouth, UK; ${ }^{7}$ Imperial College Healthcare NHS \\ Trust, London, UK, ${ }^{8}$ Health Protection Scotland, Glasgow, UK, ${ }^{9}$ Department of Health, London, UK; \\ ${ }^{10}$ Royal Free London NHS Trust, London, UK; ${ }^{11}$ University Hospitals of Leicester NHS Trust, Leicester, UK
}

Received 27 December 2013; Final revision 8 June 2014; Accepted 2 July 2014;

first published online 1 August 2014

\section{SUMMARY}

Data were extracted from the case records of UK patients admitted with laboratory-confirmed influenza $\mathrm{A}(\mathrm{H} 1 \mathrm{~N} 1)$ pdm09. White and non-White patients were characterized by age, sex, socioeconomic status, pandemic wave and indicators of pre-morbid health status. Logistic regression examined differences by ethnicity in patient characteristics, care pathway and clinical outcomes; multivariable models controlled for potential confounders. Whites $(n=630)$ and non-Whites $(n=510)$ differed by age, socioeconomic status, pandemic wave of admission, pregnancy, recorded obesity, previous and current smoking, and presence of chronic obstructive pulmonary disease. After adjustment for a priori confounders non-Whites were less likely to have received pre-admission antibiotics [adjusted odds ratio (aOR) $0.43,95 \%$ confidence interval (CI) $0 \cdot 28-0 \cdot 68, P<0 \cdot 001$ ) but more likely to receive antiviral drugs as in-patients (aOR $1 \cdot 53,95 \% \mathrm{CI}$ $1 \cdot 08-2 \cdot 18, P=0 \cdot 018$ ). However, there were no significant differences by ethnicity in delayed admission, severity at presentation for admission, or likelihood of severe outcome.

Key words: Epidemiology, influenza, influenza A, pandemic.

\section{INTRODUCTION}

Ethnicity (commonly regarded as partially synonymous with race) may evoke a strong sense of identity, unity or difference-factors inextricably linked to health. There are grounds for postulating ethnic

\footnotetext{
* Author for correspondence: Dr B. C. McKenzie, University of Nottingham, Rm C119, Clinical Sciences Building, City Hospital, Nottingham NG5 1PB, UK.

(Email: bruce.mckenzie@nottingham.ac.uk)

$\dagger$ These authors served as joint first authors.
}

differences in exposure to influenza virus, susceptibility to infection once exposed, and timely access to effective treatment [1, 2]. Disparities or inequalities in influenza-related outcomes such as pneumonia can be observed for some minority groups [3, 4]. A cross-sectional survey of 1479 US households (with oversampling of minority groups) suggests Spanishspeaking Hispanics were at increased risk of presumed influenza $\mathrm{A}(\mathrm{H} 1 \mathrm{~N} 1) \mathrm{pdm} 09$ exposure, but reduced risk of related complications; Blacks were more susceptible to self-reported complications [5]. Analysis of US 
(a)

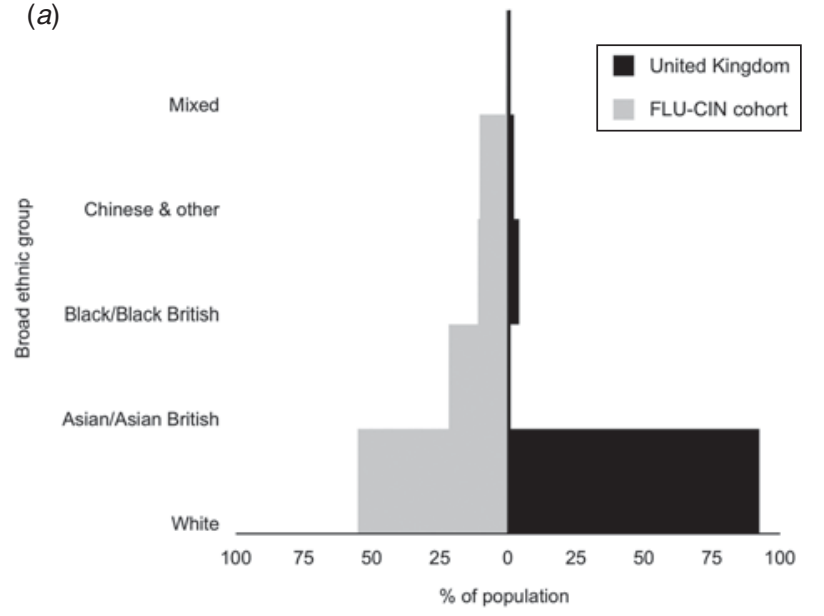

(b)

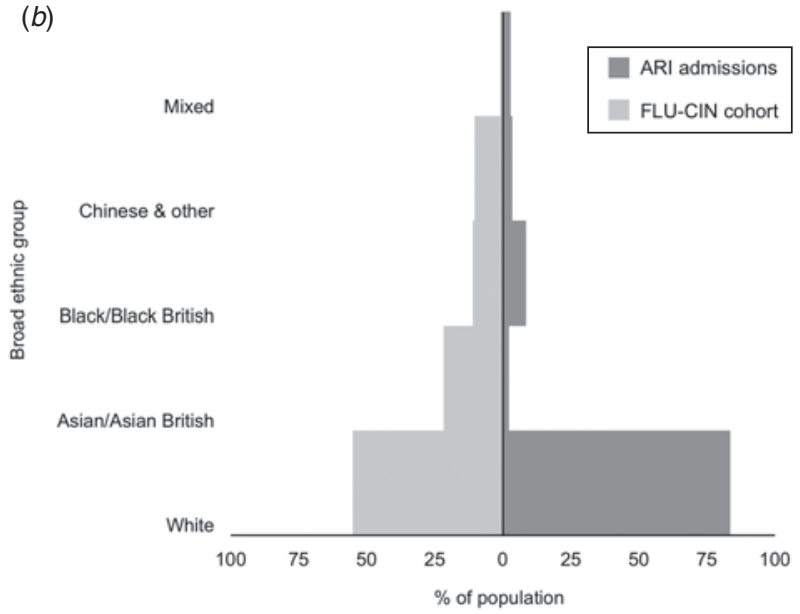

Fig. 1. FLU-CIN population pyramids for ethnic composition* with comparison to (a) the UK general population $\dagger$ and (b) admissions to UK hospitals with acute respiratory infection (ARI) in the pre-pandemic period $\$$. ${ }^{*} 1140$ cases; excludes 380 cases $(25 \%)$ missing ethnicity data. $\dagger$ Demographic data on ethnicity derived from Office of National Statistics Census (2001) and General Register Office for Scotland and Northern Ireland Statistics and Research Agency (2001). \$ Hospital Episodes Statistics data on primary discharge codes relating to possible influenza admissions (J06, J10, J11, J13-22) during November 2008-March 2009.]

Centers for Disease Control and Prevention (CDC) surveillance data collected during the 2009 pandemic found no ethnic/racial disparities in healthcare-seeking behaviour for influenza-like illness, but higher hospitalization rates in minority groups and higher paediatric mortality in Hispanics [6]. A Canadian study of 413 laboratory-confirmed influenza A(H1N1)pdm09 cases compared to test-negative community controls reports over-representation of ethnic minority cases [7].

These seemingly consistent patterns are concerning and while the effect of ethnicity may have plausible biological underpinnings it is also strongly linked to social context, requiring that international comparisons be made with caution. In the UK, based on a total of 70 paediatric deaths related to influenza A(H1N1)pdm09 (including those in the community), age-standardized mortality rates for Bangladeshi children [47 deaths per million population (dpm), 95\% confidence interval (CI) 17-103] and Pakistani children (36 dpm, 95\% CI 18-64) were found to be higher than for White British children (4 dpm, 95\% CI 3-6) [8]. Where such inequitable outcomes are suggested, our professional and moral obligations to redress them, reduce disparities in access to healthcare and improve patients' experiences can only be effected through measuring all relevant dimensions - one of which is ethnicity [9].

During the 2009 influenza pandemic the Department of Health (England) collected data on risk factors, including ethnicity, via the Influenza Clinical
Information Network (FLU-CIN) programme [10]. Analysis of the first-wave FLU-CIN cohort (MaySeptember 2009) indicated that ethnic minorities were over-represented among those admitted to hospital [10]; this was less pronounced in the second wave [11], but nevertheless raises the possibility of systematic differences in care. Figures 1 and 2, respectively, compare the ethnic composition and age profile of the FLU-CIN cohort with that of the UK general population and persons admitted to UK hospitals with acute respiratory infection (ARI) in the immediate pre-pandemic period winter season. To gain insight into these striking observations we report an analysis of FLU-CIN enhanced surveillance data over both pandemic waves, investigating possible ethnic differences in care pathway and clinical outcome in patients hospitalized with laboratoryconfirmed influenza $\mathrm{A}(\mathrm{H} 1 \mathrm{~N} 1) \mathrm{pdm} 09$ in the UK.

\section{METHODS}

As previously described, trained nursing staff extracted data from the case records of patients hospitalized for laboratory-confirmed influenza $\mathrm{A}(\mathrm{H} 1 \mathrm{~N} 1)$ pdm09, without other selection criteria [10]. These included ethnicity as recorded in case notes, according to the Office for National Statistics Census classification (www.statistics.gov.uk). This official UK system of classification can be regarded as equivalent to racial group in the majority of cases. 
(a)

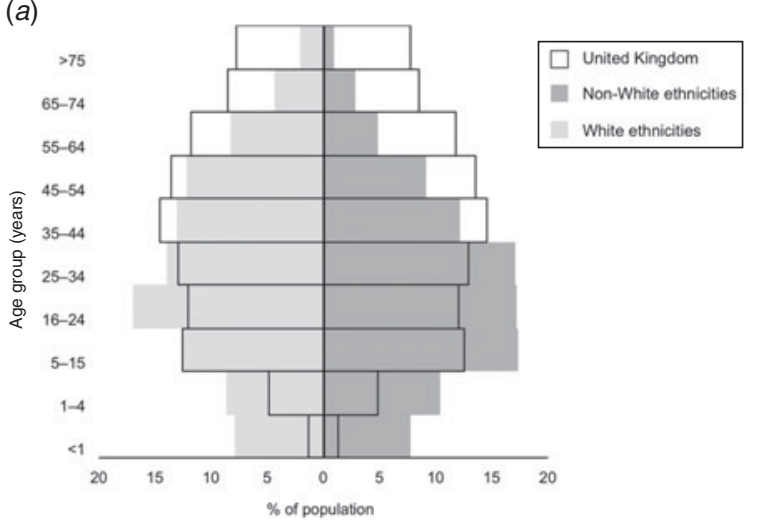

(b)

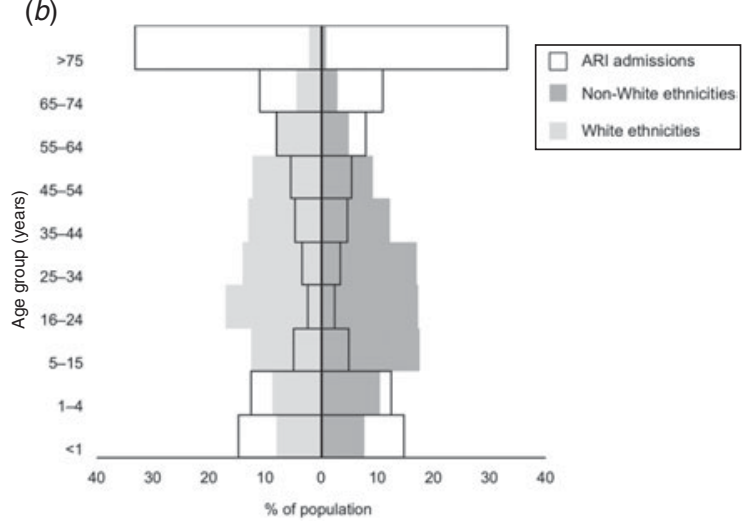

Fig. 2. FLU-CIN population pyramids for age by broad ethnic group* with comparison to (a) the UK general population $\dagger$ and $(b)$ admissions to $\mathrm{UK}$ hospitals with acute respiratory infection in the pre-pandemic period $\$$. [* 1140 cases; excludes 380 cases $(25 \%)$ missing ethnicity data. $\dagger$ Demographic data on age distribution derived from Office of National Statistics 2009 mid-year population estimates (www.statistics.gov.uk). \$ Hospital Episodes Statistics data on primary discharge codes relating to possible influenza admissions (J06, J10, J11, J13-22) during November 2008-March 2009.]

We conceptualized a patient care pathway commencing with pre-admission care in the community; primary healthcare access indicators comprised use of self-medication, general practitioner (GP) consultation and receipt of pre-admission antibiotics or antivirals. Secondary healthcare access indicators comprised two proxy measures of access to hospital: admission delay of $\geqslant 4$ days from illness onset and illness severity at presentation for admission. The pathway concluded with indicators corresponding to in-patient care, these being receipt of in-patient antibiotics or antivirals. The clinical outcomes of interest were length of stay in hospital, admission to highdependency or critical-care facilities and death. A number of covariates related to this pathway and clinical outcomes were identified and defined as follows.

Socioeconomic status was estimated from postcode of residence using the English Index of Multiple Deprivation (IMD) 2007, a composite area-based measure of deprivation that takes account of income, employment, health status and disability, education and skills, access to services, living environment and area-level crime [12]. IMD scores were grouped to facilitate comparison by 'most affluent' (IMD $\leqslant 14.999$ ), 'affluent' (IMD 15-29.999), 'deprived' (IMD 3044.999) and 'most deprived' (IMD $\geqslant 45$ ) status.

National surveillance data determined assignment to the first (pre-September 2009) or second pandemic wave [13]. The National Pandemic Flu Service (NPFS) antiviral distribution system began operation during the first wave on 23 July 2009 [14]; we created a variable representing availability of the NPFS in order to model the effect of access to the service on antiviral availability.

Baseline health status incorporated a measure of the presence of comorbidities, the Charlson Comorbidity Index (CCI); the weighted CCI scores were categorized as ' 0 ' (no comorbidities), $1-2,3-5$ and $>5$ $[15,16]$.

Early treatment with antivirals was defined as receipt within 2 days of symptom onset; late treatment was defined as receipt $>2$ days after onset of symptoms.

Delayed admission was defined by pandemic influenza experts on the FLU-CIN Steering Group as admission delay of $\geqslant 4$ days following onset of symptoms.

Illness severity at presentation was assessed using the community assessment tools (CATs) for triage (seven criteria as outlined in Supplementary Table S1), as recommended for use during a severe pandemic by the Department of Health, England $[17,18]$.

Admission for $\geqslant 2$ days was regarded as prolonged and thus a proxy measure for a more severe illness during hospitalization.

Type of admission was coded as level 0: patients whose care needs can be met through normal ward care; level 1: patients at risk of deteriorating or recently relocated from higher levels of care whose needs can be met on an acute ward with additional advice and support from the critical care team; level 2: patients requiring more detailed observation or intervention including support for a single failing organ 
system and those 'stepping down' from higher levels of care - high dependency unit (HDU); level 3: patients requiring advanced respiratory support alone or basic respiratory support together with support of at least two organ systems. This includes all complex patients requiring support for multi-organ failureintensive care unit (ICU).

Univariate logistic regression analyses (Table 1) examined unadjusted associations between ethnicity (White or non-White) and patient characteristics at the point of admission to hospital with influenza $\mathrm{A}(\mathrm{H} 1 \mathrm{~N} 1) \mathrm{pdm} 09$. A multivariable logistic regression model (Table 2) was used to adjust care pathway and clinical outcome indicators for potential confounders including age, sex, socioeconomic status, pandemic wave and (for outcomes related to in-patient care and mortality) delayed admission and severity at presentation for admission (model A). An alternative conceptual model (model B) further adjusted for variables found to be significantly maldistributed according to ethnicity (recorded obesity, current smoking and chronic obstructive pulmonary disease; pregnancy was excluded owing to the inclusion of males in the cohort).

\section{Ethical standards}

The Ethics and Confidentiality Committee (ECC) of the National Information Governance Board for Health and Social Care (NIGB) gave permission for the collection of patient-identifiable data for FLU-CIN in May 2009, noting the urgency and wider public interest of this surveillance study. Section 251 approval was not sought for exemption from gaining informed consent; provision of patient information in sentinel centres was stipulated. The authors assert that all procedures contributing to this work comply with the ethical standards of the relevant national and institutional committees on human experimentation and with the Helsinki Declaration of 1975, as revised in 2008.

\section{RESULTS}

Of 1520 admissions in the FLU-CIN cohort [11], $1140(75 \cdot 0 \%)$ had ethnicity recorded (missing data $n=380,25 \cdot 0 \%)$. A summary of patients' characteristics is given in Table 1. Non-White patients constituted almost half $(n=510,44 \cdot 7 \%)$ of the study population, the largest subgroup being Asian or Asian British $(n=249,21 \cdot 8 \%)$. Similar data for major non-White ethnic subgroups are provided in Supplementary Table S2.
Table 2 presents unadjusted (crude) odds and adjusted odds for both logistic regression models comparing non-Whites to Whites; these are grouped by care pathway and clinical outcome indicators. Similar data for major non-White ethnic subgroups are provided in Supplementary Table S3.

In respect of access to primary healthcare nonWhites were less likely to receive pre-admission antibiotics [model A, adjusted aOR (aOR) $0 \cdot 43,95 \% \mathrm{CI}$ $0 \cdot 28-0 \cdot 68, P<0 \cdot 001)$, but no more or less likely to receive pre-admission antivirals (aOR 0.86, 95\% CI $0 \cdot 51-1 \cdot 43, P=0 \cdot 554)$. Substituting the a priori variable for pandemic wave in model A for a variable representing access to the NPFS did not significantly alter the likelihood of receiving pre-admission antivirals by ethnicity (aOR $0 \cdot 89,95 \%$ CI $0.54-1 \cdot 47$, $P=0.662$ ). Furthermore, there was no significant difference in the interval between date of symptom onset and date of antiviral receipt (i.e. early vs. late treatment), by ethnicity (OR $1 \cdot 27,95 \%$ CI 0.90 $1 \cdot 77, P=0 \cdot 169)$. Insufficient data on pandemic vaccination were available for inclusion in multivariable models (missing data, $73.5 \%$ ) and 750 cases presented for admission prior to the availability of vaccine.

As a proxy measure of access to secondary healthcare, crude odds indicated non-Whites were less likely to experience an admission delay of $\geqslant 4$ days after symptom onset, but after adjustment this difference was not statistically significant. Likewise, no significant difference in illness severity at presentation for admission was observed between Whites and non-Whites (Table 2).

During the in-patient phase of the pathway nonWhite ethnic groups were more likely to receive antiviral drugs (model A: aOR 1.53, 95\% CI 1.08-2.18, $P=0.018$ ). A higher likelihood of non-Whites receiving antibiotics as in-patients became non-significant after adjustment.

In terms of clinical outcomes no significant differences were found for in-patient stays of $\geqslant 2$ days (indicating protracted illness). Crude odds indicated non-Whites were less likely to require high-dependency or intensive-care unit admission; however, after adjustment this difference was not statistically significant. No significant differences were found for mortality.

\section{DISCUSSION}

Poorer influenza-related outcomes among indigenous peoples have been observed in Alaska, Sierra Leone [19], Australia [20] and New Zealand [4, 21] during 
Table 1. FLU-CIN patients' characteristics at the point of admission to hospital with influenza A(H1N1)pdm09 in the UK by broad ethnic group $(n=1140)$

\begin{tabular}{|c|c|c|c|c|}
\hline Characteristics & $\begin{array}{l}\text { White } \\
(N=630), n(\%)\end{array}$ & $\begin{array}{l}\text { Non-White } \\
(N=510), n(\%)\end{array}$ & $\begin{array}{l}\text { Crude OR } \\
(95 \% \mathrm{CI})\end{array}$ & $P$ value \\
\hline \multicolumn{5}{|l|}{ Ethnic grouping, $N / n(\%)$} \\
\hline White & $630 / 1140(55 \cdot 3)$ & & & \\
\hline Mixed & & $11 / 1140(1 \cdot 0)$ & & \\
\hline Asian or Asian British & & $249 / 1140(21 \cdot 8)$ & & \\
\hline Black or Black British & & $129 / 1140(11 \cdot 3)$ & & \\
\hline Chinese and other & & $121 / 1140(10 \cdot 6)$ & & \\
\hline \multicolumn{5}{|l|}{ Sex } \\
\hline Male & $291(46 \cdot 2)$ & $238(46 \cdot 7)$ & $1 \cdot 00$ & \\
\hline Female & $339(53 \cdot 8)$ & $272(53 \cdot 3)$ & $0 \cdot 98(0 \cdot 78-1 \cdot 24)$ & $0 \cdot 873$ \\
\hline \multicolumn{5}{|l|}{ Age (years) } \\
\hline$<1$ & $50(7 \cdot 9)$ & $40(7 \cdot 8)$ & $1 \cdot 00$ & $P$ trend $=0.001$ \\
\hline $1-4$ & $55(8 \cdot 7)$ & $53(10 \cdot 4)$ & $1 \cdot 20(0 \cdot 69-2 \cdot 11)$ & \\
\hline $5-15$ & $79(12 \cdot 5)$ & $89(17 \cdot 5)$ & $1 \cdot 41(0 \cdot 84-2 \cdot 36)$ & \\
\hline $16-24$ & $107(17 \cdot 0)$ & $88(17 \cdot 3)$ & $1.03(0 \cdot 62-1 \cdot 70)$ & \\
\hline $25-34$ & $88(14 \cdot 0)$ & $87(17 \cdot 1)$ & $1 \cdot 24(0 \cdot 74-2 \cdot 06)$ & \\
\hline $35-44$ & $82(13 \cdot 0)$ & $62(12 \cdot 2)$ & $0.95(0 \cdot 56-1 \cdot 61)$ & \\
\hline $45-54$ & $77(12 \cdot 2)$ & $47(9 \cdot 2)$ & $0 \cdot 76(0 \cdot 44-1 \cdot 32)$ & \\
\hline $55-64$ & $52(8 \cdot 3)$ & $25(4 \cdot 9)$ & $0 \cdot 60(0 \cdot 32-1 \cdot 13)$ & \\
\hline $65-74$ & $27(4 \cdot 3)$ & $14(2 \cdot 8)$ & $0 \cdot 65(0 \cdot 30-1 \cdot 40)$ & \\
\hline$>75$ & $13(2 \cdot 1)$ & $5(1 \cdot 0)$ & $0 \cdot 48(0 \cdot 16-1 \cdot 46)$ & \\
\hline \multicolumn{5}{|l|}{ Socioeconomic status (IMD group) } \\
\hline Most affluent (IMD $\leqslant 14.999$ ) & $92(14 \cdot 6)$ & $35(6 \cdot 9)$ & $1 \cdot 00$ & $P$ trend $<0.001$ \\
\hline Affluent (IMD 15-29.999) & $126(20 \cdot 0)$ & $113(22 \cdot 2)$ & $2 \cdot 36(1 \cdot 48-3 \cdot 75)$ & \\
\hline Deprived (IMD 30-44.999) & $59(9 \cdot 4)$ & $134(26 \cdot 3)$ & $5.97(3.64-9.80)$ & \\
\hline Most deprived (IMD $\geqslant 45$ ) & $91(14 \cdot 4)$ & $143(28 \cdot 0)$ & $4 \cdot 13(2 \cdot 58-6 \cdot 61)$ & \\
\hline Missing data & $262(41 \cdot 6)$ & $85(16 \cdot 7)$ & & \\
\hline \multicolumn{5}{|l|}{ Pandemic wave } \\
\hline First wave & $180(28 \cdot 6)$ & $337(66 \cdot 1)$ & $1 \cdot 00$ & \\
\hline Second wave & $450(71 \cdot 4)$ & $173(33.9)$ & $0 \cdot 21(0 \cdot 16-0 \cdot 26)$ & $<0.001$ \\
\hline \multicolumn{5}{|l|}{ Health status } \\
\hline Current pregnancy* & $27(16 \cdot 0)$ & $39(26 \cdot 0)$ & $1 \cdot 85(1 \cdot 07-3 \cdot 20)$ & $0 \cdot 029$ \\
\hline Recorded obesity & $26(4 \cdot 1)$ & $10(2 \cdot 0)$ & $0.46(0.22-0.97)$ & $0 \cdot 042$ \\
\hline Current smoker & $120(19 \cdot 1)$ & $44(8 \cdot 6)$ & $0.45(0.31-0.66)$ & $<0.001$ \\
\hline Missing data & $220(34 \cdot 9)$ & $229(44 \cdot 9)$ & & \\
\hline Ever smoked & $171(27 \cdot 1)$ & $64(12 \cdot 5)$ & $0 \cdot 41(0 \cdot 29-0 \cdot 58)$ & $<0 \cdot 001$ \\
\hline Missing data & $220(34 \cdot 9)$ & $229(44.9)$ & & \\
\hline Asthma diagnosis & $158(25 \cdot 1)$ & $137(26 \cdot 9)$ & $1 \cdot 10(0 \cdot 84-1 \cdot 43)$ & 0.494 \\
\hline COPD diagnosis & $49(7 \cdot 8)$ & $8(1.6)$ & $0.19(0.09-0 \cdot 40)$ & $<0.001$ \\
\hline Other lung disease & $15(2 \cdot 4)$ & $13(2 \cdot 6)$ & $1 \cdot 07(0 \cdot 51-2 \cdot 27)$ & $0 \cdot 855$ \\
\hline No comorbidity, CCI 0 & $322(51 \cdot 1)$ & $299(58 \cdot 6)$ & $1 \cdot 00$ & $P$ trend $=0.052$ \\
\hline CCI 1-2 & $272(43 \cdot 2)$ & $181(35 \cdot 5)$ & $0 \cdot 72(0 \cdot 56-0 \cdot 92)$ & \\
\hline CCI 3-5 & $35(5 \cdot 6)$ & $28(5 \cdot 5)$ & $0.86(0.51-1 \cdot 45)$ & \\
\hline $\mathrm{CCI}>5$ & $1(0 \cdot 2)$ & $2(0 \cdot 4)$ & $2 \cdot 15(0 \cdot 19-23 \cdot 88)$ & \\
\hline
\end{tabular}

IMD, English Index of Multiple Deprivation (2007); COPD, chronic obstructive pulmonary disease; CCI, Charlson Comorbidity Index; OR, odds ratio; CI, confidence interval.

Percentages may not add up to 100 due to rounding; statistically significant results shown in bold $(P<0 \cdot 05)$.

* Expressed as a percentage of women of childbearing age (14-44 years).

$\dagger$ Physician-recorded in case notes. 
Table 2. Care pathway and clinical outcomes for patients admitted with influenza A(H1N1)pdm09 in the UK by broad ethnic group ( $n=1140)$

\begin{tabular}{|c|c|c|c|c|c|}
\hline Variable & $\begin{array}{l}\text { White } \\
(N=630) n(\%)\end{array}$ & $\begin{array}{l}\text { Non-White } \\
(N=510) n(\%)\end{array}$ & $\begin{array}{l}\text { Crude OR }(95 \% \mathrm{CI}) \\
P \text { value }\end{array}$ & $\begin{array}{l}\text { Adjusted OR }(95 \% \mathrm{CI}) \\
P \text { value (model A) }\end{array}$ & $\begin{array}{l}\text { Adjusted OR }(95 \% \mathrm{CI}) \\
P \text { value (model B) }\end{array}$ \\
\hline \multicolumn{6}{|c|}{ Primary healthcare access indicators } \\
\hline Self-medication & $49(7 \cdot 8)$ & $39(7 \cdot 7)$ & $0.98(0.63-1.52) ; 0.934$ & $1.33(0.75-2.35) ; 0.327$ & $1.28(0.72-2.28) ; 0.402$ \\
\hline GP consultation & $180(28 \cdot 6)$ & $139(27 \cdot 3)$ & $0.94(0.72-1.22) ; 0.622$ & $0.82(0.58-1 \cdot 15) ; 0 \cdot 255$ & $0.84(0.59-1.19) ; 0.322$ \\
\hline Missing data & $296(47 \cdot 0)$ & $227(44 \cdot 5)$ & & & \\
\hline Pre-admission antibiotic & $142(22 \cdot 5)$ & $54(10 \cdot 6)$ & $0.41(0.29-0.57) ;<0.001$ & $0.43(0.28-0.68) ;<0.001$ & $0.42(0 \cdot 27-0.67) ;<0 \cdot 001$ \\
\hline Pre-admission antiviral & $68(10 \cdot 8)$ & $51(10 \cdot 0)$ & $0.92(0.63-1.35) ; 0.663$ & $0.86(0.51-1.43) ; 0.554$ & $0 \cdot 81(0 \cdot 48-1 \cdot 35) ; 0 \cdot 412$ \\
\hline \multicolumn{6}{|c|}{ Secondary healthcare access indicators } \\
\hline Admission delay $\geqslant 4$ days & $133(21 \cdot 1)$ & $95(18 \cdot 6)$ & $0.65(0 \cdot 48-0 \cdot 88) ; 0 \cdot 006$ & $0.73(0.49-1.09) ; 0 \cdot 119$ & $0.71(0 \cdot 47-1 \cdot 07) ; 0 \cdot 103$ \\
\hline Missing data & $198(31 \cdot 4)$ & $86(16 \cdot 8)$ & & & \\
\hline Severity at presentation $\dagger$ & $468(74 \cdot 3)$ & $367(72 \cdot 0)$ & $0.89(0.68-1 \cdot 16) ; 0.378$ & $1.10(0.79-1.54) ; 0.576$ & $1 \cdot 16(0 \cdot 83-1 \cdot 64) ; 0.387$ \\
\hline \multicolumn{6}{|l|}{ In-patient care indicators } \\
\hline In-patient antibiotic & $513(81 \cdot 4)$ & $440(86 \cdot 3)$ & $1.43(1.04-1.98) ; 0.029$ & $1 \cdot 37 *(0 \cdot 84-2 \cdot 22) ; 0 \cdot 204$ & $1.46(0.94-2 \cdot 26) ; 0.095$ \\
\hline In-patient antiviral & $452(71 \cdot 8)$ & $398(78 \cdot 0)$ & $1 \cdot 40(1.07-1.84) ; 0.015$ & $1 \cdot 53^{*}(1 \cdot 08-2 \cdot 18) ; 0 \cdot 018$ & $1.66(1.15-2.39) ; 0.006$ \\
\hline \multicolumn{6}{|l|}{ Clinical outcomes } \\
\hline Length of stay $\geqslant 2$ days & $427(67 \cdot 8)$ & $357(70 \cdot 0)$ & $0 \cdot 82(0 \cdot 61-1 \cdot 11) ; 0 \cdot 195$ & $1.04 *(0.71-1.53) ; 0.840$ & $1 \cdot 11(0 \cdot 75-1 \cdot 62) ; 0.607$ \\
\hline Missing data & $93(14 \cdot 8)$ & $41(8 \cdot 0)$ & & & \\
\hline Level 2 or 3 admission & $109(17 \cdot 3)$ & $66(12 \cdot 9)$ & $0.71(0.51-0.99) ; 0.043$ & $1 \cdot 19 *(0 \cdot 74-1.92) ; 0.478$ & $1.25(0 \cdot 77-2.03) ; 0 \cdot 368$ \\
\hline Death & $35(5 \cdot 6)$ & $20(3 \cdot 9)$ & $0.69(0 \cdot 40-1 \cdot 22) ; 0.203$ & $0.80 *(0.35-1.82) ; 0.602$ & $0.77(0.33-1.80) ; 0.550$ \\
\hline
\end{tabular}

GP, General practitioner; OR, odds ratio; CI, confidence interval; adjusted OR (model A), adjusted for a priori confounders of age, sex, English Index of Multiple Deprivation (IMD 2007) score derived from postal code of residence and pandemic wave; adjusted OR (model B), adjusted for age, sex, IMD 2007 score, pandemic wave, recorded obesity, current smoking and chronic obstructive pulmonary disease.

* Adjusted for admission delay $\geqslant 4$ days and severity at presentation for admission.

$\dagger$ One or more clinical indicators of severe disease at triage (see text).

Requiring high-dependency unit or critical care unit.

Percentages may not add up to 100 due to rounding; statistically significant results shown in bold $(P<0.05)$. 
previous influenza pandemics. Similar observations were made among Canadian First Nations communities [22] and in Alaska [23] during the 2009 pandemic. By contrast, the UK's ethnic minority population is non-indigenous, and has resulted largely from immigration over the last 60 years. However, larger nonindigenous ethnic groups in North America were also reported to experience adverse outcomes during the 2009 pandemic [7, 24]. We can therefore infer complex relationships between biological and social factors that shape observed differences by ethnicity. For this reason it may not be prudent to generalize findings from one country to the ethnic minorities of another.

Measuring ethnicity accurately is a further challenge, and the value of aggregate ethnicity data in epidemiological research is contentious. Individuals may define their ethnicity in terms of parentage, race, cultural heritage or affiliation (for example). As race, skin colour, country of origin or cultural affiliation are not necessarily synonymous with a particular ethnic risk profile, there is some potential for misclassification bias. Broad groupings such as 'White' or 'non-White' encompass much diversity (in social, cultural, religious, or genetic traits for example) and sensitivities to being so 'lumped together' must be acknowledged. Such aggregation has pragmatic value (overcoming low numbers encountered with more granular ethnic groupings, where annual data cannot be pooled [25]), precedent (usage in routine statistics) and utility in hypothesis generation to scrutinize access inequalities. Unfortunately, although aggregation does improve statistical confidence, it also increases heterogeneity. As $45 \%$ of the FLU-CIN cohort was non-White, in order to balance these considerations we also compared major non-White ethnic subgroups to White groups (Supplementary Tables S2 and S3).

The pattern of over-representation of non-Whites in both the FLU-CIN cohort and the population admitted with ARI in 2008-2009 is broadly consistent (Fig. 1). ARI offers a valid comparison given that admission thresholds by ethnicity are unlikely to differ between presentation with pandemic influenza or with clinically indistinguishable respiratory virus infections (including seasonal influenza) in the previous winter season. It is equally important to note the highly significant reversal in proportion of Whites to nonWhites in FLU-CIN cases between the first and second pandemic waves (Table 1). Mapping of cases by postcode of residence according to pandemic wave (Supplementary Fig. S1) highlighted urban clustering of admissions, particularly around London and the West Midlands during the first wave, spreading to the East Midlands and Northwest (with less ethnically diverse populations) during the second wave. These data suggest that the initial predominance of nonWhite patients in the first pandemic wave and some degree of reversal as pandemic activity dispersed in the second wave was mainly attributable to place of residence, rather than inherent predisposition. This explanation would not exclude the possibility that vulnerable members of ethnic minority communities experienced higher risk of exposure to influenza $\mathrm{A}(\mathrm{H} 1 \mathrm{~N} 1) \mathrm{pdm} 09$ prior to availability of pandemic vaccine, which may still indicate the need for more effective promotion of measures such as hand hygiene advice and social distancing during the initial pandemic period.

While non-Whites were less likely to have received pre-admission antibiotics, there was no compensatory increase in the likelihood of receiving pre-admission antivirals. If antibiotic prescriptions do reflect access to primary healthcare then use of fewer antibiotics for non-Whites might indicate a reduced propensity among minority groups to seek care, culturally or educationally mediated beliefs about the value of antibiotics, or typical attitudes to risk [1,2]. Alternatively, it might indicate antibiotic prescribing practices that discriminate against ethnic minorities or are inappropriately responsive to demand from White patients. We note that Whites and non-Whites were equally likely to self-medicate; access barriers to community pharmacies may be lower. However, similar access to GP consultations and to pre-admission antivirals between Whites and non-Whites would argue against wider access barriers.

For access to secondary healthcare we found no difference between Whites and non-Whites in terms of admission delay or disease severity at presentation for admission (Table 2 and Supplementary Table S1), suggesting that differences in pre-hospital antibiotic receipt by ethnicity did not impact on timing of, or severity at admission. FLU-CIN was not configured to report influenza A(H1N1)pdm09 hospitalization rates by ethnicity for hospital catchment areas, thus our data cannot comment on excess rates observed among minority groups measured elsewhere $[6,22,24,26,27]$.

Given the absence of any difference between Whites and non-Whites at the point of admission, it is somewhat surprising that the latter were more likely to have received antiviral drugs as in-patients (Table 2). This is not explained by non-Whites presenting 
disproportionately as first-wave cases, when prescribing of antivirals could have been more cautious. The difference by ethnicity was not significant for firstwave admissions, where $74.4 \%$ of Whites $(n=134)$ and $79.0 \%$ of non-Whites $(n=266)$ received in-patient antivirals (model A: aOR $1 \cdot 23,95 \%$ CI $0 \cdot 73-2 \cdot 07, P=$ $0 \cdot 434)$. The difference was, however, significant for second-wave admissions, where $70.7 \%$ of Whites $(n=318)$ and $76 \cdot 3 \%$ of non-Whites $(n=132)$ received in-patient antivirals (model A: aOR 1.76, 95\% CI $1 \cdot 05-2 \cdot 94, P=0 \cdot 033$ ).

Our study did not find any evidence that small differences in care pathway resulted in significant differences in clinical outcome by ethnicity. Non-Whites were not disadvantaged as judged by mortality or level 2/3 admission using either our a priori or conceptual model to adjust for potential confounding. Our results juxtapose the Canadian experience where indigenous First Nations ethnicity was an independent risk factor for ICU admission [28]. The UK nonWhite population may be regarded as non-indigenous, yet mortality in New Zealand was reported as 'significantly' higher in non-indigenous Pacific Peoples (infection rate-adjusted rate ratio $3 \cdot 28,95 \%$ CI 1.44-7·49, $P$ value not stated) [27]. This may suggest that indigenous ethnic status, while being partly genetic/biological (perhaps modulating illness severity or response to therapy), is being confounded by other factors. Quinn and colleagues postulate the operation of differential risks of influenza exposure, susceptibility and healthcare access that worsen existing inequities [5], which may themselves be independently associated with ethnicity/race or socioeconomic status or both. In their review of 4874 influenza $\mathrm{A}(\mathrm{H} 1 \mathrm{~N} 1)$ pdm09-related discharges in Massachusetts, Placzek \& Madoff found both minority ethnic/racial group and lower socioeconomic status predicted ICU stay [24]. Compared to non-Hispanic Whites, Hispanics were less likely to be admitted to an ICU (aOR $0 \cdot 52,95 \%$ CI $0 \cdot 32-0 \cdot 86, P<0 \cdot 05$ ); of patients admitted to ICUs, $63 \%$ of Hispanics, $43 \%$ of non-Hispanic Blacks and only $13 \%$ of non-Hispanic Whites were among the least affluent socioeconomic group [24]. Although the authors were unable to measure differential access to healthcare, differences in risk perception, healthcare reform, cultural or language barriers are mooted as possible contributors. Taken together with our own findings, these observations likely reflect a complex and confounded relationship between clinical outcomes for influenza A(H1N1) pdm09, ethnicity and social disadvantage [29].
Our study does not provide compelling evidence of important disparities in 'downstream' access to primary or secondary care treatment for influenza A(H1N1)pdm09 in the UK. Our findings do, however, mirror the over-representation of non-Whites among A(H1N1)pdm09-related hospital admissions reported elsewhere. This might reflect a difference in susceptibility to infection, either biological or by means of difference in uptake of the 'midstream' intervention of vaccination. Our study refutes vaccination as an explanation, given that over-representation preceded availability of pandemic vaccine. Over-representation of non-Whites most likely relates to 'upstream' factors that determine exposure to the influenza virus. We provide evidence that geography played a key role in determining this pattern, which is also the basis of measuring socioeconomic status in the UK. Corelated upstream factors include population density, crowded living conditions, reliance on public transport, occupational group, acceptability of social distancing and use of communal childcare facilities $[1,2]$. The challenge for future research is to unpick the contribution of such factors and to determine whether variation is attributable to ethnicity, socioeconomic status or both.

\section{CONCLUSIONS}

Ethnicity was not a significant predictor of inequities or disparities in care pathway or of clinical outcomes for patients hospitalized with influenza $A(H 1 N 1)$ pdm09 in the UK. We did not find any evidence that small differences in care pathway resulted in significant differences in clinical outcome by ethnicity.

\section{SUPPLEMENTARY MATERIAL}

For supplementary material accompanying this paper visit http://dx.doi.org/10.1017/S0950268814001873.

\section{ACKNOWLEDGEMENTS}

We gratefully acknowledge the assistance of the many individuals, teams and organizations who came together in support of FLU-CIN; in particular Elaine Gadd and Colin Armstrong from the Department of Health, England. The contribution of S.J.B. and P.J.M.O. to the research was supported by the National Institute for Health Research (NIHR) Biomedical Research Centre based at Imperial College Healthcare NHS Trust and 
Imperial College London. Funding for FLU-CIN was provided by the Department of Health, England. The views expressed by S.J.B. and P.J.M.O. are those of the authors and not necessarily those of the National Health Service (NHS), the National Institute for Health Research (NIHR) or the Department of Health in England.

\section{DECLARATION OF INTEREST}

J.E.E. has performed consultancy for the Department of Health, England. P.R.M. has received an unrestricted educational grant from F. Hoffman-La Roche for research in the area of pandemic influenza. M.G.S. is an advisor to the Department of Health, England. K.G.N. as principal investigator of two MRC projects and one NIHR-funded project on $\mathrm{H} 5$ vaccines and 2009 H1N1 pandemic influenza vaccine has used vaccines free-of-charge from Novartis, Baxter and GSK. J.S.N.V.T. has received funding to attend influenza-related meetings, lecture and consultancy fees and research funding from several influenza antiviral drug and vaccine manufacturers [Baxter AG, GlaxoSmithKline, Novartis, F. Hoffman-La Roche, Solvay (now Abbott)]; research funding from GlaxoSmithKline and F. Hoffmann-La Roche is current and on-going, all forms of personal remuneration ceased in September 2010; he is a former employee of SmithKline Beecham plc. (now GlaxoSmithKline), Roche Products Ltd (UK) and Aventis-Pasteur MSD (now Sanofi-Pasteur MSD), all prior to 2005, with no remaining pecuniary interests by way of shareholdings, share options or accrued pension rights.

\section{REFERENCES}

1. Blumenshine $\mathbf{P}$, et al. Pandemic influenza planning in the United States from a health disparities perspective. Emerging Infectious Diseases 2008; 14: 709-715.

2. Hutchins SS, et al. Protection of racial/ethnic minority populations during an influenza pandemic. American Journal of Public Health 2009; 99 (Suppl. 2): S261-270.

3. Agency for Healthcare Research Quality. National healthcare disparities report 2011. Rockville (MD): US Department of Health and Human Services, 2012.

4. Wilson N, et al. Differential mortality rates by ethnicity in 3 influenza pandemics over a century, New Zealand. Emerging Infectious Diseases 2012; 18: 71-77.

5. Quinn SC, et al. Racial disparities in exposure, susceptibility, and access to health care in the US H1N1 influenza pandemic. American Journal of Public Health 2011; 101: 285-293.

6. Dee DL, et al. Racial and ethnic disparities in hospitalizations and deaths associated with 2009 pandemic
Influenza A (H1N1) virus infections in the United States. Annals of Epidemiology 2011; 21: 623-630.

7. Navaranjan D, et al. Ethnic disparities in acquiring 2009 pandemic H1N1 influenza: a case-control study. BMC Public Health 2014; 14: 214.

8. Sachedina N, Donaldson LJ. Paediatric mortality related to pandemic influenza A H1N1 infection in England: an observational population-based study. Lancet 2010; 376: 1846-1852.

9. Department of Health. Healthy lives, healthy people: Improving outcomes and supporting transparency; Part 1: A public health outcomes framework for England, 2013-2016. London: Department of Health, 2012.

10. Nguyen-Van-Tam JS, et al. Risk factors for hospitalisation and poor outcome with pandemic A/H1N1 influenza: United Kingdom first wave (May-September 2009). Thorax 2010; 65: 645-651.

11. Myles PR, et al. Predictors of clinical outcome in a national hospitalised cohort across both waves of the influenza A/H1N1 pandemic 2009-2010 in the UK. Thorax 2012; 67: 709-717.

12. Noble M, et al. Measuring multiple deprivation at the small-area level. Environment and Planning $A$ 2006; 38: 169-185.

13. McLean E, Pebody R. Epidemiological report of pandemic (H1N1) 2009 in the UK: April 2009-May 2010. London: Health Protection Agency, 2010.

14. Department of Health. A(H1N1) Swine Influenza: National Pandemic Flu Service launches today. In Gateway reference: 12310. London: Department of Health, 2009.

15. Charlson ME, et al. A new method of classifying prognostic comorbidity in longitudinal studies: Development and validation. Journal of Chronic Diseases 1987; 40: 373-383.

16. Deyo RA, Cherkin DC, Ciol MA. Adapting a clinical comorbidity index for use with ICD-9-CM administrative databases. Journal of Clinical Epidemiology 1992; 45: 613-619.

17. Semple MG, et al. An evaluation of community assessment tools (CATs) in predicting use of clinical interventions and severe outcomes during the A(H1N1)pdm09 pandemic. PLoS One 2013; 8: e 75384.

18. Myles PR, et al. Comparison of CATs, CURB-65 and PMEWS as triage tools in pandemic influenza admissions to UK hospitals: case control analysis using retrospective data. PLoS One 2012; 7: e 34428.

19. Crosby AW. America's Forgotten Pandemic: the Influenza of 1918. Cambridge, New York: Cambridge University Press, 2003.

20. Kelly H, Mercer G, Cheng A. Quantifying the risk of pandemic influenza in pregnancy and indigenous people in Australia in 2009. Eurosurveillance 2009; 14(50).

21. Pool DI. The effects of the 1918 pandemic of influenza on the Maori population of New Zealand. Bulletin of the History of Medicine 1973; 47: 273-281.

22. Boggild AK, et al. The impact of influenza on the Canadian First Nations. Canadian Journal of Public Health 2011; 102: 345-348. 
23. Centers for Disease Control and Prevention. Deaths related to 2009 pandemic influenza A (H1N1) among American Indian/Alaska Natives - 12 states, 2009. Morbidity and Mortality Weekly Report 2009; 58: 1341-1344.

24. Placzek H, Madoff L. Effect of race/ethnicity and socioeconomic status on pandemic H1N1-related outcomes in Massachusetts. American Journal of Public Health 2013; 104: e31-e38.

25. Office for National Statistics. Ethnic group statistics: a guide for the collection and classification of ethnicity data. Norwich: HMSO, 2003.

26. Morin C, et al. Racial/ethnic disparities in hospitalized influenza rates continue after the 2009 pandemic, Minnesota 2009-2011. International Conference on Emerging Infectious Diseases 2012, poster and oral presentation abstracts. Atlanta: Emerging Infectious Diseases, 2012.

27. Baker MG, et al. Pandemic influenza A (H1N1) infection and progression to hospitalization and death: how risk is influenced by ethnicity and socioeconomic position. International Conference on Emerging Infectious Diseases 2012, poster and oral presentation abstracts. Atlanta: Emerging Infectious Diseases, 2012.

28. Zarychanski R, et al. Correlates of severe disease in patients with 2009 pandemic influenza (H1N1) virus infection. Canadian Medical Association Journal 2010; 182: $257-264$.

29. Tricco AC, et al. Impact of $\mathrm{H} 1 \mathrm{~N} 1$ on socially disadvantaged populations: systematic review. PLoS One 2012; 7: e 39437. 